\title{
Transversal multilinear Radon-like transforms: local and global estimates
}

\author{
Jonathan Bennett, Neal Bez and Susana Gutiérrez
}

\begin{abstract}
We prove local " $L^{p}$-improving" estimates for a class of multilinear Radon-like transforms satisfying a strong transversality hypothesis. As a consequence, we obtain sharp multilinear convolution estimates for measures supported on fully transversal submanifolds of Euclidean space of arbitrary dimension. Motivated by potential applications in diffraction tomography, we also prove global estimates for the same class of Radon-like transforms under a natural homogeneity assumption.
\end{abstract}

\section{Introduction}

The main purpose of this paper is to obtain local and global estimates for a class of multilinear Radon-like transforms satisfying a transversality hypothesis.

A popular description of a Radon-like transform is a mapping $R$ of the form

$$
R f(x)=\int_{\mathbb{R}^{d}} f(y) \delta(F(y, x)) \psi(y, x) \mathrm{d} y,
$$

where $f: \mathbb{R}^{d} \rightarrow \mathbb{C}$ is a suitable test function, $x \in \mathbb{R}^{n}$, and $F: \mathbb{R}^{d} \times \mathbb{R}^{n} \rightarrow \mathbb{R}^{k}$ is a suitably smooth function which typically satisfies some nondegeneracy condition on the support of the cutoff function $\psi$. Here $d, k, n \in \mathbb{N}$ and $\delta$ denotes the Dirac delta distribution on $\mathbb{R}^{k}$. If $\nabla F$ does not vanish then $\delta \circ F$ is easily seen to be a well-defined distribution. Notice that $R f(x)$ may be interpreted as a surface integral (or "average") of $f$ over the submanifold

$$
M_{x}:=\left\{y \in \mathbb{R}^{d}: F(y, x)=0,(y, x) \in \operatorname{supp} \psi\right\},
$$

which generically has dimension $d-k$. It is natural to seek so-called " $L^{p}$-improving" properties of such transforms; that is, given $F$ find the exponents $p$ and $q$ for which $R$ extends to a bounded mapping from $L^{p}\left(\mathbb{R}^{d}\right)$ into $L^{q}\left(\mathbb{R}^{n}\right)$. There is a considerable literature on such problems which we do not discuss here, although the interested reader should consult the paper of Tao and Wright [14].

Mathematics Subject Classification (2010): Primary 44A12; Secondary 44A35.

Keywords: Multilinear forms, convolution estimates, diffraction tomography. 
A natural description of a multilinear Radon-like transform is a mapping $R$ of the form

$$
R f(x)=\int_{\mathbb{R}^{d_{1}} \times \cdots \times \mathbb{R}^{d_{m}}} f_{1}\left(y_{1}\right) \cdots f_{m}\left(y_{m}\right) \delta(F(y, x)) \psi(y, x) \mathrm{d} y,
$$

where $f=\left(f_{j}\right)_{j=1}^{m}, f_{j}: \mathbb{R}^{d_{j}} \rightarrow \mathbb{C}$ are suitable test functions, $x \in \mathbb{R}^{n}$, and $F: \mathbb{R}^{n} \times$ $\mathbb{R}^{d_{1}} \times \cdots \times \mathbb{R}^{d_{m}} \rightarrow \mathbb{R}^{k}$ is a suitably smooth function which typically satisfies some nondegeneracy conditions on the support of the cutoff function $\psi$. Again it is natural to seek $L^{p_{1}}\left(\mathbb{R}^{d_{1}}\right) \times \cdots \times L^{p_{m}}\left(\mathbb{R}^{d_{m}}\right) \rightarrow L^{q}\left(\mathbb{R}^{n}\right)$ estimates for $R$. By duality these qualities may be expressed as bounds on multilinear forms such as

$$
\int_{\mathbb{R}^{d_{1}} \times \cdots \times \mathbb{R}^{d_{m+1}}} \prod_{j=1}^{m+1} f_{j}\left(y_{j}\right) \delta(F(y)) \psi(y) \mathrm{d} y \leq C \prod_{j=1}^{m+1}\left\|f_{j}\right\|_{L^{p_{j}}\left(\mathbb{R}^{d_{j}}\right)} .
$$

Estimates of the form (1.2) arise frequently in problems in a variety of fields including geometric and harmonic analysis and dispersive PDE. Often these are manifested as certain multilinear singular convolution inequalities, which we now describe. In the work of Tao, Vargas, and Vega [13] it was shown that whenever $S_{1}$ and $S_{2}$ are transversal compact submanifolds of $\mathbb{R}^{d}$, where $d \geq 2$, which are smooth with nonvanishing Gaussian curvature, we have the estimate

$$
\left\|f_{1} \mathrm{~d} \sigma_{1} * f_{2} \mathrm{~d} \sigma_{2}\right\|_{L^{2}\left(\mathbb{R}^{d}\right)} \leq C\left\|f_{1}\right\|_{L^{\frac{4 d}{3 d-2}}\left(\mathrm{~d} \sigma_{1}\right)}\left\|f_{2}\right\|_{L^{\frac{4 d}{3 d-2}}\left(\mathrm{~d} \sigma_{2}\right)} .
$$

Here, $\mathrm{d} \sigma_{j}$ is the measure supported on $S_{j}$ given by

$$
\int_{\mathbb{R}^{d}} f(x) \mathrm{d} \sigma_{j}(x)=\int_{U_{j}} f\left(\Sigma_{j}\left(x^{\prime}\right)\right) \mathrm{d} x^{\prime},
$$

where $\Sigma_{j}: U_{j} \rightarrow \mathbb{R}^{d}$ parametrises $S_{j}$ for some compact subset $U_{j}$ of $\mathbb{R}^{d-1}$. See [9] for the case $d=3$ on which [13] built. The estimate in (1.3) was obtained in [13] from a $L^{d / d-1}\left(\mathbb{R}^{d}\right) \rightarrow L^{d}\left(\mathbb{R}^{d}\right)$ estimate on the Radon-like transform in (1.1) with $m=1$ and where $F$ satisfies a rotational curvature condition on the support of the cutoff. By Plancherel's theorem, (1.3) immediately implies a bilinear adjoint Fourier restriction estimate for transversal compact subsets of surfaces given by the graph of an elliptic phase (such as a paraboloid).

At higher levels of multilinearity, in particular when the level coincides with the ambient dimension, transversality is key and additional curvature hypotheses do not increase the $L^{p}$ improving nature of the singular convolution operation. It is known that if $S_{1}, \ldots, S_{d}$ are transversal $C^{1, \beta}$ codimension one submanifolds of $\mathbb{R}^{d}$ at the origin, $1 \leq q \leq \infty$ and $p_{j}^{\prime} \leq(d-1) q^{\prime}$, then

$$
\left\|f_{1} \mathrm{~d} \sigma_{1} * \cdots * f_{d} \mathrm{~d} \sigma_{d}\right\|_{L^{q}\left(\mathbb{R}^{d}\right)} \leq C \prod_{j=1}^{d}\left\|f_{j}\right\|_{L^{p_{j}}\left(\mathrm{~d} \sigma_{j}\right)}
$$

if each $f_{j} \in L^{p_{j}}\left(\mathrm{~d} \sigma_{j}\right)$ has support sufficiently close to the origin. This follows from [8] when $d=3$, and [4] for $d \geq 4$. In [3] the case $d=3, q=\infty$ was considered under certain scalable assumptions at each point of the hypersurfaces. 
A major goal of this paper is to provide a certain generalisation of (1.4) to transversal submanifolds of general codimension. This will be a consequence of Theorem 1.2 below concerning local multilinear Radon-like transform estimates. To be precise, let $U_{j}$ be a compact subset of $\mathbb{R}^{d_{j}}$ and let $\Sigma_{j}: U_{j} \rightarrow \mathbb{R}^{d}$ parametrise a $C^{1, \beta}$ and $d_{j}$-dimensional submanifold $S_{j}$ of $\mathbb{R}^{d}$, where $d \geq 2$ and $1 \leq j \leq m$. As above, define the associated measure $\mathrm{d} \sigma_{j}$ on $\mathbb{R}^{d}$ by

$$
\int_{\mathbb{R}^{d}} f(x) \mathrm{d} \sigma_{j}(x)=\int_{U_{j}} f\left(\Sigma_{j}(y)\right) \mathrm{d} y .
$$

We say that $S_{1}, \ldots, S_{m}$ are fully transversal at the origin if

$$
\bigoplus_{j=1}^{m} \operatorname{ker}\left(\mathrm{d} \Sigma_{j}(0)\right)^{*}=\mathbb{R}^{d}
$$

Theorem 1.1. Suppose that the submanifolds $S_{1}, \ldots, S_{m}$ are fully transversal at the origin. If $1 \leq q \leq \infty$ and $p_{j}^{\prime} \leq(m-1) q^{\prime}$, then there exists a constant $C$ such that

$$
\left\|f_{1} \mathrm{~d} \sigma_{1} * \cdots * f_{m} \mathrm{~d} \sigma_{m}\right\|_{L^{q}\left(\mathbb{R}^{d}\right)} \leq C \prod_{j=1}^{m}\left\|f_{j}\right\|_{L^{p_{j}}\left(\mathrm{~d} \sigma_{j}\right)}
$$

for all $f_{j} \in L^{p_{j}}\left(\mathrm{~d} \sigma_{j}\right)$ supported in a sufficiently small neighbourhood of the origin.

When $d_{j}=d-1$ for each $1 \leq j \leq m$, Theorem 1.1 was proved in [4]. The general case for $m=3$ was established by Bejenaru and Herr [1] (although under certain scalable assumptions over the entire patches $U_{j}$ in the spirit of [3]) using the nonlinear Brascamp-Lieb inequalities in [4]. Bejenaru and Herr succeeded in using this to obtain the local well-posedness of the three-dimensional Zakharov system in the full subcritical regime.

In Theorem 1.1, the most interesting case is where $p_{j}^{\prime}=(m-1) q^{\prime}$ for each $j$ since, of course, the remaining cases follow from Hölder's inequality. We shall discuss the optimality of the estimates given by Theorem 1.1 later in Section 3, including a justification that given, $1 \leq q \leq \infty$, the exponents $p_{j}$ satisfying $p_{j}^{\prime}=$ $(m-1) q^{\prime}$ cannot be improved.

Under the hypotheses of Theorem 1.1, taking $q=2$ and via Plancherel's theorem, we obtain the estimate

$$
\left\|\prod_{j=1}^{m} \widehat{f_{j} \mathrm{~d} \sigma_{j}}\right\|_{L^{2}\left(\mathbb{R}^{d}\right)} \leq C \prod_{j=1}^{m}\left\|f_{j}\right\|_{L^{(2 m-2)^{\prime}}\left(\mathrm{d} \sigma_{j}\right)}
$$

for all $f_{j} \in L^{(2 m-2)^{\prime}}\left(\mathrm{d} \sigma_{j}\right)$ supported in a sufficiently small neighbourhood of the origin. This estimate is, of course, a certain multilinear adjoint Fourier restriction estimate for fully transversal submanifolds of $\mathbb{R}^{d}$, and extends previous results of this nature in [8] and [4]. See [7] for further context and results. 
Notice that for the transversality assumption (1.5) to hold it is necessary that

$$
\sum_{j=1}^{m} d_{j}=(m-1) d
$$

It is also natural to assume that $m \geq 2$ and $1 \leq d_{j} \leq d-1$ for each $1 \leq j \leq m$. We shall now state our local result on Radon-like transforms, for which we shall assume the above restrictions on $d, m$ and each $d_{j}$. In order to state the theorem, we need to introduce some notation which will be adopted throughout the paper.

Let

$$
\mathcal{K}_{j}=\left\{d_{1}+\cdots+d_{j-1}+1, \ldots, d_{1}+\cdots+d_{j-1}+d_{j}\right\}
$$

for $1 \leq j \leq m$, so that the $\left(\mathcal{K}_{j}\right)_{j=1}^{m}$ partition $\{1 \ldots,(m-1) d\}$. Note that the cardinality of each $\mathcal{K}_{j}$ is $d_{j}$. In addition, it is natural to introduce some language from exterior algebra to express the nondegeneracy assumption on the mapping $F$ in the distribution $\delta \circ F$. We use the standard notation $\Lambda^{n}\left(\mathbb{R}^{d}\right)$ for the $n$th exterior algebra of $\mathbb{R}^{d}$. Also, we use $\star: \Lambda^{n}\left(\mathbb{R}^{d}\right) \rightarrow \Lambda^{d-n}\left(\mathbb{R}^{d}\right)$ for the Hodge star operator.

For a linear map $F: \mathbb{R}^{d_{1}} \times \cdots \times \mathbb{R}^{d_{m}} \rightarrow \mathbb{R}^{d}$, let $Y_{j}(F) \in \Lambda^{d_{j}}\left(\mathbb{R}^{d}\right)$ be given by

$$
Y_{j}(F)=\bigwedge_{k \in \mathcal{K}_{j}} F\left(e_{k}\right) \in \Lambda^{d_{j}}\left(\mathbb{R}^{d}\right)
$$

where $e_{k}$ denotes the $k$ th standard basis vector in $\mathbb{R}^{(m-1) d} \cong \mathbb{R}^{d_{1}} \times \cdots \times \mathbb{R}^{d_{m}}$.

We shall write $e_{k}$ for the $k$ th standard basis vector throughout; the dimension will be clear from the context. Also, with an index such as $k \in \mathcal{K}_{j}$, as in (1.8), we shall always mean that the operation is being performed as $k$ increases over $\mathcal{K}_{j}$.

Theorem 1.2. Let $\sum_{j=1}^{m} d_{j}=(m-1) d$. If $F: \mathbb{R}^{d_{1}} \times \cdots \times \mathbb{R}^{d_{m}} \rightarrow \mathbb{R}^{d}$ is $C^{1, \beta}$ in a neighbourhood of some point $y_{*} \in \mathbb{R}^{d_{1}} \times \cdots \times \mathbb{R}^{d_{m}}$ for some $\beta>0$ and

$$
\star \bigwedge_{j=1}^{m} \star Y_{j}\left(\mathrm{~d} F\left(y_{*}\right)\right) \neq 0,
$$

then there exists a neighbourhood $V$ of $y_{*}$ and a constant $C$ such that

$$
\int_{V} \prod_{j=1}^{m} f_{j}\left(y_{j}\right) \delta(F(y)) \mathrm{d} y \leq C \prod_{j=1}^{m}\left\|f_{j}\right\|_{L^{(m-1)^{\prime}}\left(\mathbb{R}^{d_{j}}\right)}
$$

for all nonnegative $f_{j} \in L^{(m-1)^{\prime}}\left(\mathbb{R}^{d_{j}}\right), 1 \leq j \leq m$.

It is important to point out the nondegeneracy hypothesis (1.9) need only be imposed on some neighbourhood of the zero set of $F$. Some further remarks concerning Theorem 1.2 are now in order.

Firstly, suppose we have a mapping $G:\left(\mathbb{R}^{d-1}\right)^{d-1} \rightarrow \mathbb{R}$ which is $C^{1, \beta}$ in a neighbourhood of a point $u_{*} \in\left(\mathbb{R}^{d-1}\right)^{d-1}$ and satisfies the nondegeneracy assumption

$$
\operatorname{det}\left(\nabla_{u_{1}} G\left(u_{*}\right) \cdots \nabla_{u_{d-1}} G\left(u_{*}\right)\right) \neq 0
$$


Then there exists a neighbourhood $V$ of $u_{*}$ and a constant $C$ such that

$$
\begin{aligned}
& \int_{V} f_{1}\left(u_{1}\right) \cdots f_{d-1}\left(u_{d-1}\right) f_{d}\left(u_{1}+\cdots\right.\left.+u_{d-1}\right) \delta(G(u)) \mathrm{d} u \\
& \leq C \prod_{j=1}^{d}\left\|f_{j}\right\|_{L^{(d-1)^{\prime}}\left(\mathbb{R}^{d-1}\right)}
\end{aligned}
$$

for all nonnegative $f_{j} \in L^{(d-1)^{\prime}}\left(\mathbb{R}^{d-1}\right)$. This was proved in [8] for $d=3$ and in [4] for $d \geq 4$, and the multilinear singular convolution estimate in (1.4) for hypersurfaces is a consequence of (1.11). Observe that (1.11) follows from Theorem 1.2 by taking $m=d, d_{j}=d-1$ for each $j$, and $F:\left(\mathbb{R}^{d-1}\right)^{d} \rightarrow \mathbb{R}^{d}$ given by

$$
F\left(y_{1}, \ldots, y_{d}\right)=\left(y_{d}-y_{d-1}-\cdots-y_{1}, G\left(y_{1}, \ldots, y_{d-1}\right)\right) .
$$

An especially elegant case of Theorem 1.2 occurs when $m=d=3$ and $d_{j}=2$ for each $j$. In this case, the nondegeneracy assumption (1.9) is simply that

$$
\operatorname{det}\left(Y_{1}\left(\mathrm{~d} F\left(y_{*}\right)\right) Y_{2}\left(\mathrm{~d} F\left(y_{*}\right)\right) Y_{3}\left(\mathrm{~d} F\left(y_{*}\right)\right)\right) \neq 0,
$$

where

$$
Y_{j}\left(\mathrm{~d} F\left(y_{*}\right)\right)=\partial_{\left(y_{j}\right)_{1}} F\left(y_{*}\right) \times \partial_{\left(y_{j}\right)_{2}} F\left(y_{*}\right) .
$$

Then Theorem 1.2 tells us that there exists a neighbourhood $V$ of $y_{*}$ in $\left(\mathbb{R}^{2}\right)^{3}$ and a constant $C$ such that

$$
\int_{V} f_{1}\left(y_{1}\right) f_{2}\left(y_{2}\right) f_{3}\left(y_{3}\right) \delta(F(y)) \mathrm{d} y \leq C\left\|f_{1}\right\|_{L^{2}\left(\mathbb{R}^{2}\right)}\left\|f_{2}\right\|_{L^{2}\left(\mathbb{R}^{2}\right)}\left\|f_{3}\right\|_{L^{2}\left(\mathbb{R}^{2}\right)}
$$

for all nonnegative $f_{1}, f_{2}, f_{3} \in L^{2}\left(\mathbb{R}^{2}\right)$.

One interpretation of inequality (1.10) is that it is a distributional $L^{p}$ variant of the multilinear weighted $L^{2}$ estimates of Tao [12]. At the end of this section we provide another more explicit perspective from a dispersive PDE point of view.

Next, we present our global extension of Theorem 1.2 under the additional hypothesis that $F$ is homogeneous of degree one. Our original motivation for considering this setting stemmed from the appearance of certain globally defined multilinear Radon-like transforms in diffraction tomography, and we shall elaborate on this shortly. The hypothesis that $F$ be homogeneous of degree one is of course a natural assumption since it encompasses the case of linear $F$. Observe that mappings $F$ which are homogeneous of degree one are not, in general, smooth at the origin. As a consequence, the neighbourhood $V$ obtained from Theorem 1.2 will not contain the origin and thus a trivial globalisation argument based on a direct isotropic scaling argument will not run.

Theorem 1.3. Suppose $\sum_{j=1}^{m} d_{j}=(m-1) d$. If $F: \mathbb{R}^{d_{1}} \times \cdots \times \mathbb{R}^{d_{m}} \rightarrow \mathbb{R}^{d}$ is homogeneous of degree one, with regularity $C^{1, \beta}$ for some $\beta>0$, and

$$
\star \bigwedge_{j=1}^{m} \star Y_{j}(\mathrm{~d} F(\omega)) \neq 0
$$


for each $\omega$ in the unit sphere in $\mathbb{R}^{d_{1}} \times \cdots \times \mathbb{R}^{d_{m}}$, then there exists a constant $C$ such that

$$
\int_{\mathbb{R}^{d_{1} \times \cdots \times \mathbb{R}^{d_{m}}}} \prod_{j=1}^{m} f_{j}\left(y_{j}\right) \delta(F(y)) \mathrm{d} y \leq C \prod_{j=1}^{m}\left\|f_{j}\right\|_{L^{(m-1)^{\prime}}\left(\mathbb{R}^{d_{j}}\right)}
$$

for all nonnegative $f_{j} \in L^{(m-1)^{\prime}}\left(\mathbb{R}^{d_{j}}\right), 1 \leq j \leq m$.

There is a superficially stronger version of Theorem 1.3 where the hypothesis (1.13) is made on the support of a homogeneous degree zero cutoff function $\psi$, and the resulting inequality is replaced by

$$
\int_{\mathbb{R}^{d_{1}} \times \cdots \times \mathbb{R}^{d_{m}}} \prod_{j=1}^{m} f_{j}\left(y_{j}\right) \delta(F(y)) \psi(y) \mathrm{d} y \leq C \prod_{j=1}^{m}\left\|f_{j}\right\|_{L^{(m-1)^{\prime}}\left(\mathbb{R}^{d_{j}}\right)} .
$$

This fact, which is immediately apparent from the forthcoming proof, allows Theorem 1.3 to be applied in situations where the form in (1.13) has vanishing points. Indeed our argument yields a more quantitative result where the hypothesis (1.13) is replaced with

$$
\left|\star \bigwedge_{j=1}^{m} \star Y_{j}(\mathrm{~d} F(\omega))\right| \geq \varepsilon
$$

on the support of the cutoff function $\psi$. In this situation, as may be readily observed from our arguments, we may conclude that

$$
\int_{\mathbb{R}^{d_{1} \times \cdots \times \mathbb{R}^{d_{m}}}} \prod_{j=1}^{m} f_{j}\left(y_{j}\right) \delta(F(y)) \psi(y) \mathrm{d} y \leq C \varepsilon^{-\gamma} \prod_{j=1}^{m}\left\|f_{j}\right\|_{L^{(m-1)^{\prime}}\left(\mathbb{R}^{d_{j}}\right)}
$$

for some positive exponent $\gamma$. The value of $\gamma$ that our argument produces is unfortunately quite far from optimal; a change of variables argument involving linear $F$ shows that $\gamma$ cannot be smaller than $1 /(m-1)$.

Again, we emphasise that (1.13) (or (1.14)) only need hold for each unit vector $\omega$ which belongs to some neighbourhood of the zero set of $F$. We can see the importance of this in the example $F:\left(\mathbb{R}^{2}\right)^{3} \rightarrow \mathbb{R}^{3}$ given by

$$
F\left(y_{1}, y_{2}, y_{3}\right)=\left(y_{3}-y_{1}-y_{2},\left|y_{1}\right|-\left|y_{2}\right|\right)
$$

since although the modulus function ceases to be smooth at the origin, $F$ is smooth at unit vectors $\omega$ belonging to a small neighbourhood of the zero set of $F$.

Similar explicit examples of globally defined multilinear Radon-like transforms arise in diffraction tomography, and it was these specific operators that inspired Theorem 1.3. A simple example arises in the theory of obstacle scattering, and in particular in the recovery of singularities (in the scale of classical $L^{2}$ Sobolev spaces) of a potential $q$ by its so-called Born approximation $q_{B}$ from backscattering data. As may be seen (for example in [10]), smoothing estimates for the 
mapping $q \mapsto q-q_{B}$ may be reduced to bounds on certain multilinear operators comprising the associated Born series ${ }^{1}$. Of particular relevance in [10] are smoothing estimates for the second term of the Born series, and in particular of its so-called "spherical part", $S(q)$, defined by

$$
\widehat{S(q)}(x)=\frac{i \pi}{|x|} \int_{\Gamma(x)} \widehat{q}(x-y) \widehat{q}(y) \mathrm{d} \sigma_{x}(y),
$$

where ${ }^{\wedge}$ denotes the $n$-dimensional Fourier transform, $\Gamma(x)$ the sphere centered at $x / 2$ and radius $|x| / 2$ in $\mathbb{R}^{n}$, and $\mathrm{d} \sigma_{x}$ the arclength measure on $\Gamma(x)$. Crucial to the results in [10] is the establishment of $\dot{H}^{s_{1}} \times \dot{H}^{s_{2}} \rightarrow \dot{H}^{s_{3}}$ bounds for $S(q)$ in three dimensions, for certain $s_{1}, s_{2}, s_{3} \in \mathbb{R}$; here $\dot{H}^{s}$ denotes the usual homogeneous $L^{2}$ Sobolev space of order $s$. From the definition of the $\dot{H}^{s}$ norm, this bound is quickly recast as an $L^{2} \times L^{2} \rightarrow L^{2}$ bound on the bilinear operator

$$
L_{s_{1}, s_{2}, 1-s_{3}}(f, g)(x)=\frac{1}{|x|^{1-s_{3}}} \int_{\Gamma(x)} \frac{f(y)}{|y|^{s_{1}}} \frac{g(x-y)}{|x-y|^{s_{2}}} \mathrm{~d} \sigma_{x}(y) .
$$

By duality, this $L^{2}$ bound may then be further recast as an $L^{2} \times L^{2} \times L^{2}$ bound on the trilinear form

$$
\Lambda_{a, b, c}(f, g, h)=\int_{\left(\mathbb{R}^{2}\right)^{3}} \frac{f(y)}{|y|^{a}} \frac{g(z)}{|z|^{b}} \frac{h(x)}{|x|^{c}} \delta(F(x, y, z)) \mathrm{d} x \mathrm{~d} y \mathrm{~d} z,
$$

where $F:\left(\mathbb{R}^{2}\right)^{3} \longrightarrow \mathbb{R}^{3}$ is given by

$$
F(x, y, z)=\left(x-y-z,\left|y-\frac{x}{2}\right|-\left|\frac{x}{2}\right|\right) .
$$

Here we have set $a=s_{1}, b=s_{2}$ and $c=1-s_{3}$. A straightforward calculation shows that in this particular case the left-hand side of (1.13) or (1.14) is given by

$$
\left|\star \bigwedge_{j=1}^{m} \star Y_{j}(\mathrm{~d} F(x, y, z))\right|=\frac{\left|x \cdot(y-x / 2)^{\perp}\right|}{2|x||y-x / 2|}=\frac{1}{2}|\sin (\theta(x, y-x / 2))|
$$

on the zero set of $F$, where $y^{\perp}=\left(-y_{2}, y_{1}\right)$ and $\theta(x, y)$ denotes the angle between two vectors $x, y \in \mathbb{R}^{2}$. The form in (1.17) resembles those appearing in our global multilinear Radon-like transform result (Theorem 1.3), with the exception of the powers of $|x|,|y|$ and $|z|$ that feature and the fact that the crucial quantity in (1.18) fails to be bounded below. However, a suitable decomposition away from the vanishing set of (1.18) allows us to express (1.17) as a sum of multilinear Radon-like transforms which do fall into the class that we consider in this paper. More specifically, if $\left\{\psi_{j}\right\}_{j \geq 0}$ is a partition of unity on $\left(\mathbb{R}^{2}\right)^{3} \backslash\{0\}$ with each $\psi_{j}$ homogeneous of degree zero and adapted to the set $\Gamma_{j}=\{(x, y, z) \in$ $\left.\left(\mathbb{R}^{2}\right)^{3}:|\sin (\theta(x, y-x / 2))| \sim 2^{-j}\right\}$, then on $\{F=0\} \cap \Gamma_{j}$ it is straightforward to

\footnotetext{
${ }^{1}$ We refer the reader to [10] and the references there for the relevant background to this singularity recovery problem, including the definition of the Born series and approximation.
} 
observe that $|y|^{a}|z|^{b}|x|^{c} \sim 2^{-a j}|x|^{a+b+c}$ and, by construction, the form in (1.18) is bounded below by a constant multiple of $2^{-j}$. Setting $a+b+c=0$ yields $\Lambda_{a, b, c}=\sum_{j} \Lambda_{a, b, c}^{(j)}$, where

$$
\Lambda_{a, b, c}^{(j)}(f, g, h) \sim 2^{a j} \int_{\left(\mathbb{R}^{2}\right)^{3}} f(y) g(z) h(x) \delta(F(x, y, z)) \psi_{j}(x, y, z) \mathrm{d} x \mathrm{~d} y \mathrm{~d} z
$$

The forms $\Lambda_{a, b, c}^{(j)}$ are indeed of the type that we consider in this paper, and we may apply the estimate (1.15) with $\varepsilon \sim 2^{-j}$ to obtain

$$
\Lambda_{a, b, c}(f, g, h) \lesssim \sum_{j=0}^{\infty} 2^{\gamma j} 2^{a j}\|f\|_{2}\|g\|_{2}\|h\|_{2},
$$

which clearly has the desired bound provided $\gamma+a<0$. Of course the size of $\gamma$ is decisive here. If we could take $\gamma=1 / 2$ in (1.15) we would recover the smoothing result of Ruiz and Vargas [10] (involving a gain of almost half a derivative in $\left.q \mapsto q-q_{B}\right)$. For general $\gamma$ we obtain a gain of almost $1-\gamma$ derivatives, which of course only has content for $\gamma<1$. Unfortunately the methods of this paper, which are based on the local results of [4], generate still larger values of $\gamma$. Despite this rather negative conclusion, our very general Theorem 1.3 does have the virtue of shedding some light on the geometry of the underlying multilinear forms in this scattering problem. The inefficiencies in our arguments that lead to the suboptimal exponent $\gamma$ in the global inequality (1.15) are an unfortunate feature of our globalisation method, since the quantitative version of the local Theorem 1.2 (see the forthcoming Theorem 2.1) does indeed carry the optimal exponent $\gamma=1 /(m-1)$. Indeed, PDE applications of this more effective local theorem have been successfully found by Bejenaru and Herr in the setting of the Zahkarov system [1].

Our proof of the local Theorem 1.2 rests on the nonlinear Brascamp-Lieb inequalities proved in [4], and proceeds via a parametrisation of the support of the distribution $\delta \circ F$. Using this approach, one is led estimates of the form

$$
\int_{U} \prod_{j=1}^{m} f_{j}\left(B_{j}(x)\right) \mathrm{d} x \leq C \prod_{j=1}^{m}\left\|f_{j}\right\|_{L^{p_{j}}\left(\mathbb{R}^{d_{j}}\right)},
$$

where $U$ is a neighbourhood of $\mathbb{R}^{d}$, and $B_{j}: U \rightarrow \mathbb{R}^{d_{j}}$ are local submersions. See Section 2 for a precise statement of the inequalities from [4] that we need in this paper. We also note that $L^{p}$-improving estimates of the type (1.20) have been obtained by Tao and Wright [14] for $m=2$, and Stovall [11] for $m \geq 3$; in these works, curvature plays a more prominent role than transversality, and are restricted to the case where the fibres of the underlying mappings $B_{j}$ are one-dimensional.

We provide a direct proof of Theorem 1.3 in Section 4. We note that one may also obtain Theorem 1.3 as a consequence of the global nonlinear BrascampLieb inequalities in the companion paper [5]. In the setting of estimates of the form (1.20), our globalisation argument is especially natural.

To conclude this section, we mention an interpretation of some of our results from a dispersive PDE point of view. For $n \in \mathbb{N}$, consider solutions $u_{j}: \mathbb{R} \times \mathbb{R}^{n} \rightarrow \mathbb{R}$ 
to the dispersive PDE

$$
\partial_{t} u_{j}(t, x)=i h_{j}(D) u_{j}(t, x)
$$

for $1 \leq j \leq n+1$. Here, $D$ is the frequency operator $i^{-1} \nabla$ and the $h_{j}: \mathbb{R}^{n} \rightarrow \mathbb{R}$ are the dispersion relations. If the initial data are sufficiently nice (say Schwartz functions) we may write the solutions as

$$
u_{j}(t, x)=\int_{\mathbb{R}^{n}} \widehat{u_{j}(0)}(\xi) e^{2 \pi i\left(t h_{j}(\xi)+x \cdot \xi\right)} \mathrm{d} \xi,
$$

and consequently

$$
\int_{\mathbb{R} \times \mathbb{R}^{n}} \prod_{j=1}^{n+1} u_{j}(t, x) \mathrm{d} t \mathrm{~d} x=\int_{\left(\mathbb{R}^{n}\right)^{n+1}} \prod_{j=1}^{n+1} \widehat{u_{j}(0)}\left(\xi_{j}\right) \delta(F(\xi)) \mathrm{d} \xi
$$

where $F:\left(\mathbb{R}^{n}\right)^{n+1} \rightarrow \mathbb{R}^{n+1}$ is given by

$$
F(\xi)=\left(\xi_{1}+\cdots+\xi_{n+1}, h_{1}\left(\xi_{1}\right)+\cdots+h_{n+1}\left(\xi_{n+1}\right)\right) .
$$

In this case, the nondegeneracy condition (1.9) is equivalent to the transversality of the hypersurfaces in $\mathbb{R}^{n+1}$ which are the graphs of the $h_{j}$. If this holds then from either Theorem 1.2 or Theorem 1.3 we obtain "interaction" estimates of the type

$$
\left|\int_{V \subseteq \mathbb{R} \times \mathbb{R}^{n}} \prod_{j=1}^{n+1} u_{j}(t, x) \mathrm{d} t \mathrm{~d} x\right| \leq C \prod_{j=1}^{n+1}\left\|\widehat{u_{j}(0)}\right\|_{L^{n^{\prime}\left(\mathbb{R}^{n}\right)}} .
$$

When $n=2,(1.22)$ can be interpreted as a certain weak form of the sharp trilinear Fourier extension inequality from [7]. In the case of $n+1$ solutions of the classical Schrödinger equation, where $h_{j}(\xi)=-|\xi|^{2}$ for each $j$, we have the appropriate transversality by insisting that the initial data have Fourier transforms whose supports do not simultaneously meet any affine hyperplane. When each $h_{j}(\xi)=|\xi|$ (strictly speaking, giving rise to a pseudo-differential operator associated to the wave equation), by Theorem 1.3 we may take $V=\mathbb{R} \times \mathbb{R}^{n}$ if we have initial data whose Fourier transforms are supported in appropriate conical regions.

Finally, we mention the case where $h_{j}(\xi)=-v_{j} \cdot \xi$ for some fixed $v_{j} \in \mathbb{R}^{n}$, in which case (1.21) is of course a transport equation. When the vectors $v_{1}, \ldots, v_{n+1}$ are non-coplanar we obtain global estimates in (1.22). Although the exponents in (1.22) continue to be optimal in this setting, we note that for $n \geq 3$ the stronger estimate

$$
\left|\int_{\mathbb{R} \times \mathbb{R}^{n}} \prod_{j=1}^{n+1} u_{j}(t, x) \mathrm{d} t \mathrm{~d} x\right| \leq C \prod_{j=1}^{n+1}\left\|u_{j}(0)\right\|_{L^{n}\left(\mathbb{R}^{n}\right)}
$$

is true, with (1.22) following by an application of the Hausdorff-Young inequality on the right-hand side of (1.23). Since $u_{j}(t, x)=u_{j}(0)\left(x-v_{j} t\right)$, the inequality (1.23) is simply a reinterpretation of the classical Loomis-Whitney inequality. 
This last observation suggests that inequality (1.10) in Theorem 1.2 may be strengthened for $m \geq 3$ to

$$
\int_{V} \prod_{j=1}^{m} f_{j}\left(y_{j}\right) \delta(F(y)) \mathrm{d} y \leq C \prod_{j=1}^{m}\left\|\widehat{f}_{j}\right\|_{L^{m-1}\left(\mathbb{R}^{d_{j}}\right)} .
$$

Organisation of the paper. In Section 2 we prove a certain quantitative version of Theorem 1.2. In Section 3 we deduce the multilinear singular convolution estimates in Theorem 1.1. Finally, Theorem 1.3 is proved in Section 4.

Acknowledgement. We would like to thank Juan Antonio Barceló and Alberto Ruiz for drawing our attention to the form (1.17), and to Juan Antonio Barceló for his significant contribution during the early stages of this work. We are also grateful for the support of the Isaac Newton Institute for Mathematical Sciences where part of this work was carried out.

\section{The local case}

We begin with a quantitative version of Theorem 1.2 which extends earlier versions in [8] and [4], and from which we deduce Theorem 1.1 in Section 3.

Theorem 2.1. Let $\beta, \varepsilon, \kappa>0$ be given and suppose $\sum_{j=1}^{m} d_{j}=(m-1) d$. If $F: \mathbb{R}^{d_{1}} \times \cdots \times \mathbb{R}^{d_{m}} \rightarrow \mathbb{R}^{d}$ is such that $\|F\|_{C^{1, \beta}} \leq \kappa$ in a neighbourhood of some point $y_{*} \in \mathbb{R}^{d_{1}} \times \cdots \times \mathbb{R}^{d_{m}}$ and

$$
\left|\star \bigwedge_{j=1}^{m} \star Y_{j}\left(\mathrm{~d} F\left(y_{*}\right)\right)\right| \geq \varepsilon
$$

then there exists a neighbourhood $V$ of $y_{*}$, depending only on $\beta, \varepsilon, \kappa$ and $d$, and a constant $C$ depending only on $d$, such that

$$
\int_{V} \prod_{j=1}^{m} f_{j}\left(y_{j}\right) \delta(F(y)) \mathrm{d} y \leq C \varepsilon^{-1 /(m-1)} \prod_{j=1}^{m}\left\|f_{j}\right\|_{L^{(m-1)^{\prime}}\left(\mathbb{R}^{d_{j}}\right)}
$$

for all nonnegative $f_{j} \in L^{(m-1)^{\prime}}\left(\mathbb{R}^{d_{j}}\right), 1 \leq j \leq m$.

It will be clear from the proof of Theorem 2.1 that the rate of blow-up $\varepsilon^{-1 /(m-1)}$ is sharp.

Before beginning the proof of Theorem 2.1 we offer an outline in the case explicitly presented in the introduction, where $m=d=3$ and each $d_{j}=2$. For additional simplicity, set $y_{*}=0$. The first step is to reduce to a "canonical" mapping $F$ via linear changes of variables. Specifically, there exist invertible matrices $M$ and $N$ such that if

$$
\widetilde{F}:=N \circ F \circ M^{-1}
$$


then

$$
\mathrm{d} \widetilde{F}(0)=\left(\begin{array}{llllll}
1 & 0 & 0 & 1 & 0 & 0 \\
0 & 1 & 0 & 0 & 1 & 0 \\
0 & 0 & 1 & 0 & 0 & 1
\end{array}\right)
$$

To see this, let $n_{1}, n_{2}, n_{3} \in \mathbb{S}^{2}$ be given by

$$
n_{1}=\frac{Y_{3}(\mathrm{~d} F(0))}{\left|Y_{3}(\mathrm{~d} F(0))\right|}, \quad n_{2}=\frac{Y_{2}(\mathrm{~d} F(0))}{\left|Y_{2}(\mathrm{~d} F(0))\right|}, \quad n_{3}=\frac{Y_{1}(\mathrm{~d} F(0))}{\left|Y_{1}(\mathrm{~d} F(0))\right|},
$$

and let $N$ be the matrix whose $i$ th row is equal to $n_{i}$. Furthermore, let $N_{1}, N_{2}$ and $N_{3}$ be the matrices given by

$$
N_{1}=\left(\begin{array}{c}
n_{1} \\
n_{2}
\end{array}\right), \quad N_{2}=\left(\begin{array}{c}
n_{1} \\
n_{3}
\end{array}\right), \quad N_{3}=\left(\begin{array}{c}
n_{2} \\
n_{3}
\end{array}\right)
$$

and let $F_{1}, F_{2}$ and $F_{3}$ be the matrices given by

$$
F_{j}=\left(\begin{array}{ll}
\partial_{\left(y_{j}\right)_{1}} F(0) & \partial_{\left(y_{j}\right)_{2}} F(0)
\end{array}\right)
$$

for $j=1,2,3$. Finally, we let $M$ be the block diagonal matrix given by

$$
M=\left(\begin{array}{ccc}
E_{1} N_{1} F_{1} & 0 & 0 \\
0 & E_{2} N_{2} F_{2} & 0 \\
0 & 0 & E_{3} N_{3} F_{3}
\end{array}\right),
$$

where $E_{1}$ and $E_{3}$ are simply the identity matrices, and $E_{2}$ is the elementary matrix which reflects in the line spanned by $(1,1)$. It is easy to verify that the above construction yields (2.1). Observe that using the scalar quadruple product formula, one obtains

$$
\left|\operatorname{det}\left(N_{j} F_{j}\right)\right|=\left|Y_{j}(\mathrm{~d} F(0))\right||\operatorname{det}(N)|
$$

for each $j=1,2,3$, which allows one to obtain the desired quantitative control.

Note that if $F$ were the linear map given by (2.1) then, by parametrising the zero set of $F$, one is led to linear mappings $B_{j}: \mathbb{R}^{3} \rightarrow \mathbb{R}^{2}$ whose kernels are one-dimensional and constitute the coordinate axes. An appropriate form of the implicit function theorem allows one to handle small nonlinear perturbations of this situation, and the required multilinear Radon transform estimate follows in this simple situation from the nonlinear Loomis-Whitney inequality in [8]. At this stage, in the general case, we rely on the following nonlinear Brascamp-Lieb inequalities.

Theorem 2.2 ([4]). Suppose that for each $1 \leq j \leq m$ the mappings $B_{j}: \mathbb{R}^{d} \rightarrow \mathbb{R}^{d_{j}}$ are $C^{1, \beta}$ submersions in a neighbourhood of a point $x_{0} \in \mathbb{R}^{d}$. Suppose further that

$$
\bigoplus_{j=1}^{m} \operatorname{ker} \mathrm{d} B_{j}\left(x_{0}\right)=\mathbb{R}^{d}
$$


Then there exists a neighbourhood $U$ of $x_{0}$ and a constant $C$ such that

$$
\int_{U} \prod_{j=1}^{m} f_{j} \circ B_{j} \leq C \prod_{j=1}^{m}\left\|f_{j}\right\|_{L^{m-1}\left(\mathbb{R}^{d_{j}}\right)}
$$

for all nonnegative $f_{j} \in L^{m-1}\left(\mathbb{R}^{d_{j}}\right), 1 \leq j \leq m$.

However, for most cases, the kernels of the derivatives of the mappings $B_{j}$ at the point in question do not form a direct sum decomposition. So, as a cautionary note, in general, a further "tensoring" argument is needed at this stage in order to apply Theorem 2.2. For this to proceed, we combine the $B_{j}$ appropriately in "block" form, to give rise to mappings which do satisfy the direct sum hypotheses of Theorem 2.2 (see [4] for the origin of this idea).

Proof of Theorem 2.1. We have $\sum_{j=1}^{m} d_{j}=(m-1) d$, in which case we frequently identify $\left(y_{1}, \ldots, y_{m}\right) \in \mathbb{R}^{d_{1}} \times \cdots \times \mathbb{R}^{d_{m}}$ with $\left(u_{1}, \ldots, u_{(m-1) d}\right) \in \mathbb{R}^{(m-1) d}$.

We first prove that the following special case implies Theorem 2.1.

Proposition 2.3. Let $\beta, \kappa>0$ be given and suppose $\sum_{j=1}^{m} d_{j}=(m-1) d$. If $F: \mathbb{R}^{d_{1}} \times \cdots \times \mathbb{R}^{d_{m}} \rightarrow \mathbb{R}^{d}$ is such that $\|F\|_{C^{1, \beta}} \leq \kappa$ in a neighbourhood of some point $y_{*} \in \mathbb{R}^{d_{1}} \times \cdots \times \mathbb{R}^{d_{m}}$, and $\mathrm{d} F\left(y_{*}\right): \mathbb{R}^{d_{1}} \times \cdots \times \mathbb{R}^{d_{m}} \rightarrow \mathbb{R}^{d}$ is given by

$$
\left(\mathrm{d} F\left(y_{*}\right) u\right)_{i}=\sum_{j=0}^{m-2} u_{i+j d}
$$

for $1 \leq i \leq d$, then there exists a neighbourhood $V$ of $y_{*}$, depending only on $\beta, \kappa$ and $d$, and a constant $C$ depending only on $d$, such that

$$
\int_{V} \prod_{j=1}^{m} f_{j}\left(u_{j}\right) \delta(F(u)) \mathrm{d} u \leq C \prod_{j=1}^{m}\left\|f_{j}\right\|_{L^{(m-1)^{\prime}\left(\mathbb{R}^{d_{j}}\right)}}
$$

for all nonnegative $f_{j} \in L^{(m-1)^{\prime}}\left(\mathbb{R}^{d_{j}}\right), 1 \leq j \leq m$.

We remark that if $F$ satisfies (2.4) then $\mathrm{d} F\left(y_{*}\right)$ is the augmented matrix comprising $m-1$ copies of the identity matrix $I_{d}$, and moreover $\left|\star \bigwedge_{j=1}^{m} \star Y_{j}\left(\mathrm{~d} F\left(y_{*}\right)\right)\right|=1$.

The proof that Proposition 2.3 implies Theorem 2.1 is based on the change of variables outlined above. To set this up in general, for $1 \leq j \leq m$, we let

$$
\mathcal{K}_{j}^{*}=\left\{d-\left(d_{1}^{*}+\cdots+d_{j-1}^{*}+d_{j}^{*}\right)+1, \ldots, d-\left(d_{1}^{*}+\cdots+d_{j-1}^{*}\right)\right\},
$$

where $d_{j}^{*}:=d-d_{j}$. Clearly, $\mathcal{K}_{j}^{*}$ has $d_{j}^{*}$ elements.

Now let $\left\{n_{k}: k \in \mathcal{K}_{j}^{*}\right\}$ be any orthonormal basis for the orthogonal complement of the space spanned by $\left\{\mathrm{d} F\left(y_{*}\right)\left(e_{k}\right): k \in \mathcal{K}_{j}\right\}$, and let $N$ be the $d \times d$ matrix whose $i$ th row is equal to $n_{i}$ for each $1 \leq i \leq d$.

Denote by $N_{j}$ the $d_{j} \times d$ matrix obtained by deleting from $N$ the rows $n_{k}$ for each $k \in \mathcal{K}_{j}^{*}$, and by $F_{j}$ the $d \times d_{j}$ matrix obtained by deleting the $k$ th column from $\mathrm{d} F\left(y_{*}\right)$ for $k \notin \mathcal{K}_{j}$. 
From Lemma A.1, for each $1 \leq j \leq m$, we have

$$
\star Y_{j}\left(\mathrm{~d} F\left(y_{*}\right)\right)=\left\|Y_{j}\left(\mathrm{~d} F\left(y_{*}\right)\right)\right\|_{\Lambda^{d_{j}}\left(\mathbb{R}^{d}\right)} \bigwedge_{k \in \mathcal{K}_{j}^{*}} n_{k}
$$

We shall use (2.5) to demonstrate various identities involving the determinants of the matrices describing the changes of variables, from which we obtain inequalities which allow us to establish the claimed dependencies on the sizes of the neighbourhoods and the constants arising in Theorem 2.1.

It follows from (2.5) that

$$
\left|\star \bigwedge_{j=1}^{m} \star Y_{j}\left(\mathrm{~d} F\left(y_{*}\right)\right)\right|=\left|\star \bigwedge_{j=1}^{m} \bigwedge_{k \in \mathcal{K}_{j}^{*}} n_{k}\right| \prod_{j=1}^{m}\left\|Y_{j}\left(\mathrm{~d} F\left(y_{*}\right)\right)\right\|_{\Lambda^{d_{j}}\left(\mathbb{R}^{d}\right)}
$$

and therefore

$$
\left|\star \bigwedge_{j=1}^{m} \star Y_{j}\left(\mathrm{~d} F\left(y_{*}\right)\right)\right|=|\operatorname{det}(N)| \prod_{j=1}^{m}\left\|Y_{j}\left(\mathrm{~d} F\left(y_{*}\right)\right)\right\|_{\Lambda^{d_{j}\left(\mathbb{R}^{d}\right)}} .
$$

Furthermore,

$$
\begin{aligned}
\left|\operatorname{det}\left(N_{j} F_{j}\right)\right| & =\left|\left\langle\bigwedge_{k \notin \mathcal{K}_{j}^{*}} n_{k}, \bigwedge_{\ell \in \mathcal{K}_{j}} \mathrm{~d} F\left(y_{*}\right)\left(e_{\ell}\right)\right\rangle_{\Lambda^{d_{j}\left(\mathbb{R}^{d}\right)}}\right| \\
& =\left|\left\langle\bigwedge_{k \notin \mathcal{K}_{j}^{*}} a_{k}, Y_{j}\left(\mathrm{~d} F\left(y_{*}\right)\right)\right\rangle_{\Lambda^{d_{j}\left(\mathbb{R}^{d}\right)}}\right| \\
& =\left\|Y_{j}\left(\mathrm{~d} F\left(y_{*}\right)\right)\right\|_{\Lambda^{d_{j}}\left(\mathbb{R}^{d}\right)}\left|\left\langle\bigwedge_{k \notin \mathcal{K}_{j}^{*}} n_{k}, \star \bigwedge_{\ell \in \mathcal{K}_{j}^{*}} n_{\ell}\right\rangle_{\Lambda^{d_{j}}\left(\mathbb{R}^{d}\right)}\right| \\
& =\left\|Y_{j}\left(\mathrm{~d} F\left(y_{*}\right)\right)\right\|_{\Lambda^{d_{j}\left(\mathbb{R}^{d}\right)}}|\operatorname{det}(N)| .
\end{aligned}
$$

Consequently,

$$
\prod_{j=1}^{m}\left|\operatorname{det}\left(N_{j} F_{j}\right)\right|=|\operatorname{det}(N)|^{m-1}\left|\star \bigwedge_{j=1}^{m} \star Y_{j}\left(\mathrm{~d} F\left(y_{*}\right)\right)\right| .
$$

Now define $M$ to be the block diagonal $\sum_{j=1}^{m} d_{j} \times \sum_{j=1}^{m} d_{j}$ matrix with $d_{j} \times d_{j}$ invertible matrices $M_{j}$ on the diagonal. Here $M_{j}$ is given by

$$
M_{j}=E_{j}^{-1} A_{j} F_{j}
$$

where $E_{j}: \mathbb{R}^{d_{j}} \rightarrow \mathbb{R}^{d_{j}}$ is given by

$$
E_{j}\left(x_{1}, \ldots, x_{d_{j}}\right)=\left(x_{d_{1}^{\prime}+\cdots+d_{j-1}^{\prime}+1}, \ldots, x_{d_{j}}, x_{1}, \ldots, x_{d_{1}^{\prime}+\cdots+d_{j-1}^{\prime}}\right)
$$

for $2 \leq j \leq m-1, E_{1}=I_{d_{1}}$ and $E_{m}=I_{d_{m}}$. Since $\sum_{\ell=1}^{m} d_{\ell}^{\prime}=d$ and each $d_{\ell}^{\prime} \geq 1$ it follows that

$$
\sum_{\ell=1}^{j-1} d_{\ell}<(j-1) d<\sum_{\ell=1}^{j} d_{\ell}
$$


for each $2 \leq j \leq m-1$; in particular, it follows that each $E_{j}$ is well defined. By construction, we have that if $\widetilde{F}: \mathbb{R}^{d_{1}} \times \cdots \times \mathbb{R}^{d_{m}} \rightarrow \mathbb{R}^{d}$ is the map given by

$$
\widetilde{F}:=N \circ F \circ M^{-1},
$$

then $\mathrm{d} \widetilde{F}\left(\widetilde{y}_{*}\right): \mathbb{R}^{d_{1}} \times \cdots \times \mathbb{R}^{d_{m}} \rightarrow \mathbb{R}^{d}$ is the map given by

$$
\left(\mathrm{d} \widetilde{F}\left(\widetilde{y}_{*}\right) u\right)_{i}=\sum_{j=0}^{m-2} u_{i+j d}
$$

for $1 \leq i \leq d$, where $\widetilde{y}_{*}:=M y_{*}$.

By changes of variables,

$$
\int_{V} \prod_{j=1}^{m} f_{j}\left(y_{j}\right) \delta(F(y)) \mathrm{d} y=\frac{|\operatorname{det}(N)|}{|\operatorname{det}(M)|} \int_{M(V)} \prod_{j=1}^{m}\left(f_{j} \circ M_{j}^{-1}\right)\left(y_{j}\right) \delta(\widetilde{F}(y)) \mathrm{d} y,
$$

where the neighbourhood $V$ of $y_{*}$ shall be chosen momentarily. It follows from (2.6) that $|\operatorname{det}(N)|$ is bounded below by a constant depending only on $\varepsilon, \kappa$ and $d$. Since $|\operatorname{det}(M)|=\prod_{j=1}^{m}\left|\operatorname{det}\left(M_{j}\right)\right|$ and $\left|\operatorname{det}\left(M_{j}\right)\right|=\left|\operatorname{det}\left(N_{j} F_{j}\right)\right|$ it follows from $(2.7)$ that

$$
|\operatorname{det}(M)|=|\operatorname{det}(N)|^{m-1}\left|\star \bigwedge_{j=1}^{m} \star Y_{j}\left(\mathrm{~d} F\left(y_{*}\right)\right)\right|
$$

and $|\operatorname{det}(M)|$ is also bounded below by a constant depending only on $\varepsilon, \kappa$ and $d$. Therefore, the operator norm of $M^{-1}$ and, consequently, the $C^{1, \beta}$ norm of $\widetilde{F}$ are bounded above by a constant depending only on $\beta, \varepsilon, \kappa$ and $d$. By Proposition 2.3 there exists a neighbourhood $V$, depending only on $\beta, \varepsilon, \kappa$ and $d$, and a constant $C$, depending only on $d$, such that

$$
\int_{M(V)} \prod_{j=1}^{m}\left(f_{j} \circ M_{j}^{-1}\right)\left(y_{j}\right) \delta(\widetilde{F}(y)) \mathrm{d} y \leq C \prod_{j=1}^{m}\left\|f_{j} \circ M_{j}^{-1}\right\|_{L^{(m-1)^{\prime}\left(\mathbb{R}^{d_{j}}\right)}} .
$$

Therefore,

$$
\begin{aligned}
\int_{V} \prod_{j=1}^{m} f_{j}\left(u_{j}\right) \delta(F(y)) \mathrm{d} y & \leq C \frac{|\operatorname{det}(N)|}{|\operatorname{det}(M)|}\left(\prod_{j=1}^{m}\left|\operatorname{det}\left(M_{j}\right)\right|\right)^{1 /(m-1)^{\prime}} \prod_{j=1}^{m}\left\|f_{j}\right\|_{L^{(m-1)^{\prime}}\left(\mathbb{R}^{d_{j}}\right)} \\
& \leq C \varepsilon^{-1 /(m-1)} \prod_{j=1}^{m}\left\|f_{j}\right\|_{L^{(m-1)^{\prime}\left(\mathbb{R}^{d_{j}}\right)}}
\end{aligned}
$$

The second inequality follows from (2.8). This concludes our proof that Theorem 2.1 is implied by Proposition 2.3.

Proof of Proposition 2.3. Without loss of generality we suppose that $y_{*}=0$ and $F(0)=0$. Since

$$
\left.\mathrm{d} F(0)\right|_{\operatorname{span}\left\{e_{r}:(m-2) d+1 \leq r \leq(m-1) d\right\}}=I_{d},
$$


we obtain from Theorem B.1 that there exists a neighbourhood $W$ of the origin in $\mathbb{R}^{(m-2) d}$ and a mapping $\eta: W \rightarrow \mathbb{R}^{d}$ such that, for each

$$
x=\left(u_{1}, \ldots, u_{m-2},\left(u_{m-1}\right)_{1}, \ldots,\left(u_{m-1}\right)_{d_{m-1}-d_{m}^{\prime}}\right) \in W,
$$

we have $F(x, \eta(x))=0$ and $\eta(0)=0$. The neighbourhood $W$ depends only on $\beta, \kappa$ and $d$, and the mapping $\eta$ satisfies $\|\eta\|_{C^{1, \beta}} \leq \widetilde{\kappa}$ for some constant $\widetilde{\kappa}$ which depends only on $\beta, \kappa$ and $d$.

Let $B_{j}: W \subset \mathbb{R}^{(m-2) d} \rightarrow \mathbb{R}^{d_{j}}$ be the map given by

$$
B_{j}(x)=\left(x_{1+\sum_{\ell=1}^{j-1} d_{\ell}}, x_{2+\sum_{\ell=1}^{j-1} d_{\ell}}, \ldots, x_{\sum_{\ell=1}^{j} d_{\ell}}\right)
$$

for $1 \leq j \leq m-2$,

$$
B_{m-1}(x)=\left(x_{1+\sum_{\ell=1}^{m-2} d_{\ell}}, \ldots, x_{(m-2) d},-\eta_{1}(x), \ldots,-\eta_{d_{m}^{\prime}}(x)\right)
$$

and

$$
B_{m}(x)=\left(-\eta_{d_{m}^{\prime}+1}(x), \ldots,-\eta_{d}(x)\right) .
$$

Let $S^{(j)}$ denote the $(m-2)$-tuple obtained by deleting $j-2(\bmod m)$ and $j-1(\bmod m)$ from the $m$-tuple $(1, \ldots, m)$. Then define $B_{j}^{\oplus}: W \subset \mathbb{R}^{(m-2) d} \rightarrow \mathbb{R}^{d_{j}^{\oplus}}$, where

$$
d_{j}^{\oplus}=\sum_{\ell=1}^{m-2} d_{S_{\ell}^{(j)}}
$$

by

$$
B_{j}^{\oplus}(x)=\left(B_{S_{1}^{(j)}}(x), \ldots, B_{S_{m-2}^{(j)}}(x)\right) .
$$

Lemma 2.4. Suppose $3 \leq j \leq m-1$. Then

$$
\begin{aligned}
& \operatorname{ker} \mathrm{d} B_{1}^{\oplus}(0)=\operatorname{span}\left\{e_{r}: r=1+\sum_{\ell=1}^{m-2} d_{\ell}, \ldots,(m-2) d\right\}, \\
& \operatorname{kerd} B_{2}^{\oplus}(0)=\operatorname{span}\left\{e_{r}: r=d_{m}^{\prime}+1, \ldots, d_{1}\right\}, \\
& \operatorname{kerd} B_{j}^{\oplus}(0)=\operatorname{span}\left\{e_{r}-e_{r+d}: r=1+\sum_{\ell=1}^{j-3} d_{\ell}, \ldots,\left(\sum_{\ell=1}^{j-1} d_{\ell}\right)-d\right\}, \\
& \operatorname{kerd} B_{m}^{\oplus}(0)=\operatorname{span}\left\{e_{r}-e_{r+d}: r=1+\sum_{\ell=1}^{m-3} d_{\ell}, \ldots,(m-3) d\right\} \\
& \quad+\operatorname{span}\left\{e_{s}: s=(m-3) d+1, \ldots, d_{m}^{\prime}+(m-3) d\right\} .
\end{aligned}
$$

Proof. Of course, for $1 \leq j \leq m-2, B_{j}$ is linear and therefore $\mathrm{d} B_{j}(0)=B_{j}$. By the chain rule,

$$
\mathrm{d} \eta(0)=-\left.\mathrm{d} F(0)\right|_{\operatorname{span}\left\{e_{r}: 1 \leq r \leq(m-2) d\right\}}
$$

because $\left.\mathrm{d} F(0)\right|_{\operatorname{span}\left\{e_{r}:(m-2) d+1 \leq r \leq(m-1) d\right\}}=I_{d}$. Hence,

$$
\mathrm{d} B_{m-1}(0) x=\left(x_{1+\sum_{\ell=1}^{m-2} d_{\ell}}, \ldots, x_{(m-2) d},-\sum_{\ell=0}^{m-3} x_{1+\ell d}, \ldots,-\sum_{\ell=0}^{m-3} x_{d_{m}^{\prime}+\ell d}\right)
$$


and

$$
\mathrm{d} B_{m}(0) x=\left(-\sum_{\ell=0}^{m-3} x_{d_{m}^{\prime}+1+\ell d}, \ldots,-\sum_{\ell=0}^{m-3} x_{d+\ell d}\right) .
$$

The claimed expression for the kernel of each $\mathrm{d} B_{j}^{\oplus}(0)$ now follows from the fact that

$$
\operatorname{ker} \mathrm{d} B_{j}^{\oplus}(0)=\bigcap_{\ell=1}^{m-2} \operatorname{ker} \mathrm{d} B_{S_{\ell}^{(j)}}(0)
$$

and straightforward considerations.

Corollary 2.5. We have

$$
\bigoplus_{j=1}^{m} \operatorname{kerd} B_{j}^{\oplus}(0)=\mathbb{R}^{(m-2) d}
$$

Proof. For $3 \leq j \leq m-1$, define

$$
\begin{aligned}
\mathfrak{B}_{1} & :=\left\{e_{r}: r=1+\sum_{\ell=1}^{m-2} d_{\ell}, \ldots,(m-2) d\right\} \\
\mathfrak{B}_{2} & :=\left\{e_{r}: r=d_{m}^{\prime}+1, \ldots, d_{1}\right\} \\
\mathfrak{B}_{j} & :=\left\{e_{r}-e_{r+d}: r=1+\sum_{\ell=1}^{j-3} d_{\ell}, \ldots,\left(\sum_{\ell=1}^{j-1} d_{\ell}\right)-d\right\} \\
\mathfrak{B}_{m} & :=\left\{e_{r}-e_{r+d}: r=1+\sum_{\ell=1}^{m-3} d_{\ell}, \ldots,(m-3) d\right\} \\
\cup & \left.\cup e_{s}: s=(m-3) d+1, \ldots, d_{m}^{\prime}+(m-3) d\right\},
\end{aligned}
$$

and define

$$
\mathfrak{B}=\bigcup_{j=1}^{m} \mathfrak{B}_{j}
$$

Since $\sum_{j=1}^{m}\left|\mathfrak{B}_{j}\right|=(m-2) d$ it suffices to check that $\operatorname{span}(\mathfrak{B})=\mathbb{R}^{(m-2) d}$. In order to show this, we prove that $e_{r} \in \operatorname{span}(\mathfrak{B})$ for each $r \in\{1, \ldots,(m-2) d\}$. To help clarify the notation in the rest of the proof, note that

$$
\{1, \ldots,(m-2) d\}=\bigcup_{j=2}^{m-1}\left\{1+\sum_{\ell=1}^{j-2} d_{\ell}, \ldots, \sum_{\ell=1}^{j-1} d_{\ell}\right\} \bigcup\left\{1+\sum_{\ell=1}^{m-2} d_{\ell}, \ldots,(m-2) d\right\}
$$

and in our considerations we split

$$
\begin{aligned}
\left\{1+\sum_{\ell=1}^{j-2} d_{\ell}, \ldots, \sum_{\ell=1}^{j-1} d_{\ell}\right\}=\left\{1+\sum_{\ell=1}^{j-2} d_{\ell}, \ldots, d_{m}^{\prime}+(j-2) d\right\} & \\
& \cup\left\{d_{m}^{\prime}+1+(j-2) d, \ldots, \sum_{\ell=1}^{j-1} d_{\ell}\right\} .
\end{aligned}
$$


Case 1: $r \in\left\{1+\sum_{\ell=1}^{m-3} d_{\ell}, \ldots, d_{m}^{\prime}+(m-3) d\right\}$.

Clearly we have $e_{r} \in \mathfrak{B}_{m} \subseteq \operatorname{span}(\mathfrak{B})$ for $r \in\left\{(m-3) d+1, \ldots, d_{m}^{\prime}+(m-3) d\right\}$. For the remaining $r \in\left\{1+\sum_{\ell=1}^{m-3} d_{\ell}, \ldots,(m-3) d\right\}$ we have $r+d \in\{1+$ $\left.\sum_{\ell=1}^{m-2} d_{\ell}, \ldots,(m-2) d\right\}$, so

$$
e_{r}=\left(e_{r}-e_{r+d}\right)+e_{r+d} \in \mathfrak{B}_{m}+\mathfrak{B}_{1} \subseteq \operatorname{span}(\mathfrak{B}) .
$$

Case 2: $r \in\left\{1+\sum_{\ell=1}^{j-2} d_{\ell}, \ldots, d_{m}^{\prime}+(j-2) d\right\}, 2 \leq j \leq m-2$.

Write

$$
e_{r}=\sum_{\ell=1}^{m-j-1}\left(e_{r+(\ell-1) d}-e_{r+\ell d}\right)+e_{r+(m-j-1) d} .
$$

We have

$$
r+(\ell-1) d \geq 1+(\ell-1) d+\sum_{k=1}^{j-2} d_{k} \geq 1+\sum_{k=1}^{j+\ell-3} d_{k}
$$

and

$$
r+(\ell-1) d \leq d_{m}^{\prime}+(j+\ell-3) d \leq\left(\sum_{k=1}^{j+\ell-1} d_{k}\right)-d .
$$

For each $1 \leq \ell \leq m-j-1$ we have $2 \leq j \leq \ell+j-1 \leq m-2$, so

$$
e_{r+(\ell-1) d}-e_{r+\ell d} \in \mathfrak{B}_{j+\ell} \text {. }
$$

Similarly, we have $r+(m-j-1) d \in\left\{1+\sum_{\ell=1}^{m-3} d_{\ell}, \ldots, d_{m}^{\prime}+(m-3) d\right\}$ and therefore $e_{r+(m-j-1) d} \in \operatorname{span}(\mathfrak{B})$ by case 1 . Hence $e_{r} \in \operatorname{span}(\mathfrak{B})$ for $r \in\{1+$ $\left.\sum_{\ell=1}^{j-2} d_{\ell}, \ldots, d_{m}^{\prime}+(j-2) d\right\}$, where $2 \leq j \leq m-2$.

Case 3: $r \in\left\{d_{m}^{\prime}+1, \ldots, d_{1}\right\}$.

Immediately we have $e_{r} \in \mathfrak{B}_{2} \subseteq \operatorname{span}(\mathfrak{B})$ for such $r$.

Case 4: $r \in\left\{d_{m}^{\prime}+1+(j-2) d, \ldots, \sum_{\ell=1}^{j-1} d_{\ell}\right\}, 3 \leq j \leq m-1$.

Write

$$
e_{r}=\sum_{\ell=1}^{j-2}\left(e_{r-(\ell-1) d}-e_{r-\ell d}\right)+e_{r-(j-2) d}
$$

Since

$$
r-\ell d \in\left\{1+\sum_{k=1}^{j-\ell-2} d_{k}, \ldots,\left(\sum_{k=1}^{j-\ell} d_{k}\right)-d\right\}
$$

and $r-(j-2) d \in\left\{d_{m}^{\prime}+1, \ldots, d_{1}\right\}$, we get $e_{r-(\ell-1) d}-e_{r-\ell d} \in \mathfrak{B}_{j-\ell-1}$ and, by case 3 , $e_{r-(j-2) d} \in \mathfrak{B}_{2}$. Hence, $e_{r} \in \operatorname{span}(\mathfrak{B})$ for $r \in\left\{d_{m}^{\prime}+1+(j-2) d, \ldots, \sum_{\ell=1}^{j-1} d_{\ell}\right\}$ and each $2 \leq j \leq m-1$.

Case 5: $r \in\left\{1+\sum_{\ell=1}^{m-2}, \ldots,(m-2) d\right\}$.

Immediately we have $e_{r} \in \mathfrak{B}_{1}$ for such $r$. This completes our proof of Corollary 2.5 . 
Given Corollary 2.5 we can apply Theorem 1.3 of [4], a certain quantitative version of Theorem 2.2 , to obtain a neighbourhood $U$ of the origin in $\mathbb{R}^{(m-2) d}$, depending on $\beta, \kappa$ and $d$, and a constant $C$, depending on $d$, such that

$$
\int_{U} \prod_{j=1}^{m} g_{j}\left(B_{j}^{\oplus}(x)\right) \mathrm{d} x \leq C \prod_{j=1}^{m}\left\|g_{j}\right\|_{L^{m-1}\left(\mathbb{R}^{d_{j}^{\oplus}}\right)}
$$

for all $g_{j} \in L^{m-1}\left(\mathbb{R}^{d_{j}^{\oplus}}\right)$. Given nonnegative $f_{j} \in L^{(m-1)^{\prime}}\left(\mathbb{R}^{d_{j}}\right)$ we let

$$
f_{j}^{\otimes}=\bigotimes_{\ell=1}^{m-2} f_{S_{\ell}^{(j)}}^{1 /(m-2)}
$$

so that

$$
\int_{U} \prod_{j=1}^{m} f_{j}^{\otimes}\left(B_{j}^{\oplus}(x)\right) \mathrm{d} x=\int_{U} \prod_{j=1}^{m} f_{j}\left(B_{j}(x)\right) \mathrm{d} x
$$

and

$$
\prod_{j=1}^{m}\left\|f_{j}^{\otimes}\right\|_{L^{m-1}\left(\mathbb{R}^{\left.d_{j}^{\oplus}\right)}\right.}=\prod_{j=1}^{m}\left\|f_{j}\right\|_{L^{(m-1)^{\prime}}\left(\mathbb{R}^{d_{j}}\right)},
$$

and therefore

$$
\int_{U} \prod_{j=1}^{m} f_{j}\left(B_{j}(x)\right) \mathrm{d} x \leq C \prod_{j=1}^{m}\left\|f_{j}\right\|_{L^{(m-1)^{\prime}}\left(\mathbb{R}^{d_{j}}\right)} .
$$

Finally, since $F(x, \eta(x))=0$ for $x \in W \subset \mathbb{R}^{(m-2) d}$,

$$
\left.\mathrm{d} F(0)\right|_{\operatorname{span}\left\{e_{r}:(m-2) d+1 \leq r \leq(m-1) d\right\}}=I_{d},
$$

and $\|F\|_{C^{1, \beta}} \leq \kappa$, we obtain a neighbourhood $V \subset \mathbb{R}^{(m-1) d}$, depending on $\beta, \kappa$ and $d$, such that

$$
\int_{V} \prod_{j=1}^{m} f_{j}\left(u_{j}\right) \delta(F(u)) \mathrm{d} u \leq 2 \int_{U} \prod_{j=1}^{m} f_{j}\left(B_{j}(x)\right) \mathrm{d} x .
$$

This completes the proof of Proposition 2.3.

Proposition 2.3 implies Theorem 2.1 and thus our proof of Theorem 2.1 is complete.

\section{Multilinear singular convolution}

In this section, we prove Theorem 1.1 and demonstrate that the exponents are sharp.

Proof of Theorem 1.1. Recall that it suffices to handle the case where $p_{j}^{\prime}=(m-1) q^{\prime}$ for each $1 \leq j \leq m$. Furthermore, since the operator

$$
\left(f_{1}, \ldots, f_{m}\right) \mapsto f_{1} \mathrm{~d} \sigma_{1} * \cdots * f_{m} \mathrm{~d} \sigma_{m}
$$


is trivially bounded as a mapping $L^{1}\left(\mathrm{~d} \sigma_{1}\right) \times \cdots \times L^{1}\left(\mathrm{~d} \sigma_{m}\right) \rightarrow L^{1}\left(\mathbb{R}^{d}\right)$, using multilinear interpolation, it suffices to handle the case $(p, q)=\left((m-1)^{\prime}, \infty\right)$.

If $x \in \mathbb{R}^{d}$ then

$$
\begin{aligned}
f_{1} \mathrm{~d} \sigma_{1} * \cdots & * f_{m} \mathrm{~d} \sigma_{m}(x) \\
& =\int_{\mathbb{R}^{d_{1}} \times \cdots \times \mathbb{R}^{d_{m}}} \prod_{j=1}^{m} f_{j}\left(\Sigma_{j}\left(y_{j}\right)\right) \delta\left(\Sigma_{1}\left(y_{1}\right)+\cdots+\Sigma_{m}\left(y_{m}\right)-x\right) \mathrm{d} y \\
& =\int_{\mathbb{R}^{d_{1}} \times \cdots \times \mathbb{R}^{d_{m}}} \prod_{j=1}^{m} g_{j}\left(y_{j}\right) \delta(F(y)) \mathrm{d} y,
\end{aligned}
$$

where, of course, $g_{j}=f_{j} \circ \Sigma_{j}$ and

$$
F(y)=\Sigma_{1}\left(y_{1}\right)+\cdots+\Sigma_{m}\left(y_{m}\right)-x .
$$

As a matrix, $\mathrm{d} F(0)$ is the augmented matrix comprised of $\mathrm{d} \Sigma_{1}(0), \ldots, \mathrm{d} \Sigma_{m}(0)$, and therefore

$$
Y_{j}(\mathrm{~d} F(0))=\bigwedge_{k=1}^{d_{j}} \mathrm{~d} \Sigma_{j}(0)\left(e_{k}\right)
$$

If we take any orthonormal basis $\left\{n_{k}: k \in \mathcal{K}_{j}^{*}\right\}$ for the orthogonal complement of the image of $\mathrm{d} \Sigma_{j}(0)$, or equivalently for $\operatorname{ker}\left(\mathrm{d} \Sigma_{j}(0)\right)^{*}$, then

$$
\star Y_{j}(\mathrm{~d} F(0))=\left\|Y_{j}(\mathrm{~d} F(0))\right\|_{\Lambda^{d_{j}}\left(\mathbb{R}^{d}\right)} \bigwedge_{k \in \mathcal{K}_{j}^{*}} n_{k}
$$

by Lemma A.1. Consequently,

$$
\star \bigwedge_{j=1}^{m} \star Y_{j}(\mathrm{~d} F(0))=\prod_{j=1}^{m}\left\|Y_{j}(\mathrm{~d} F(0))\right\|_{\Lambda^{d_{j}}\left(\mathbb{R}^{d}\right)} \operatorname{det}\left(n_{1} \cdots n_{d}\right)
$$

and by (1.5) this quantity is nonzero. Since $F \in C^{1, \beta}$ uniformly in $x$ belonging to a sufficiently small neighbourhood of the origin, by Theorem 2.1, it follows that there is some constant $C$ such that

$$
\left\|f_{1} \mathrm{~d} \sigma_{1} * \cdots * f_{m} \mathrm{~d} \sigma_{m}\right\|_{L^{\infty}\left(\mathbb{R}^{d}\right)} \leq C \prod_{j=1}^{m}\left\|f_{j}\right\|_{L^{(m-1)^{\prime}}\left(\mathrm{d} \sigma_{j}\right)}
$$

if the support of each $f_{j} \in L^{(m-1)^{\prime}}\left(\mathrm{d} \sigma_{j}\right)$ is sufficiently close to the origin. This completes the proof when $(p, q)=\left((m-1)^{\prime}, \infty\right)$ and hence the proof of Theorem 1.1 entirely.

To see that the exponents are sharp, suppose, setting a contradiction, that when $1 \leq q \leq \infty$ and for some $p^{\prime}>(m-1) q^{\prime}$ there exists a constant $C$ such that

$$
\left\|f_{1} \mathrm{~d} \sigma_{1} * \cdots * f_{m} \mathrm{~d} \sigma_{m}\right\|_{L^{q}\left(\mathbb{R}^{d}\right)} \leq C \prod_{j=1}^{m}\left\|f_{j}\right\|_{L^{p}\left(\mathrm{~d} \sigma_{j}\right)}
$$


for all $f_{j} \in L^{p}\left(\mathrm{~d} \sigma_{j}\right)$. It follows in particular that

$$
\left\|f_{1} \mathrm{~d} \sigma_{1} * \cdots * f_{m} \mathrm{~d} \sigma_{m}\right\|_{L^{2}\left(\mathbb{R}^{d}\right)} \leq C \prod_{j=1}^{m}\left\|f_{j}\right\|_{L^{r}\left(\mathrm{~d} \sigma_{j}\right)}
$$

for all $f_{j} \in L^{r}\left(\mathrm{~d} \sigma_{j}\right)$, where $r<(2 m-2)^{\prime}$. To see this, interpolate (3.1) with the $L^{1}\left(\mathrm{~d} \sigma_{1}\right) \times \cdots \times L^{1}\left(\mathrm{~d} \sigma_{m}\right) \rightarrow L^{1}\left(\mathbb{R}^{d}\right)$ estimate if $q>2$, or with the $L^{(m-1)^{\prime}}\left(\mathrm{d} \sigma_{1}\right) \times$ $\cdots \times L^{(m-1)^{\prime}}\left(\mathrm{d} \sigma_{m}\right) \rightarrow L^{\infty}\left(\mathbb{R}^{d}\right)$ estimate if $q<2$. To see that (3.2) is false, it suffices to consider the case where the mappings $\Sigma_{j}: \mathbb{R}^{d_{j}} \rightarrow \mathbb{R}^{d}$ are linear and thus

$$
\bigoplus_{j=1}^{m} \operatorname{ker} \Sigma_{j}^{*}=\mathbb{R}^{d}
$$

Setting $g_{j}=f_{j} \circ \Sigma_{j}$, by Plancherel's theorem and the linearity of the $\Sigma_{j}$ we have

$$
\left\|f_{1} \mathrm{~d} \sigma_{1} * \cdots * f_{m} \mathrm{~d} \sigma_{m}\right\|_{L^{2}\left(\mathbb{R}^{d}\right)}^{2}=\int_{\mathbb{R}^{d}} \prod_{j=1}^{m}\left|\widehat{g}_{j}\right|^{2}\left(\Sigma_{j}^{*} x\right) \mathrm{d} x
$$

and therefore (3.2) is equivalent to

$$
\int_{\mathbb{R}^{d}} \prod_{j=1}^{m}\left|\widehat{g_{j}}\right|^{2}\left(\Sigma_{j}^{*} x\right) \mathrm{d} x \leq C \prod_{j=1}^{m}\left\|g_{j}\right\|_{L^{r}\left(\mathbb{R}^{d_{j}}\right)}^{2}
$$

for all $g_{j} \in L^{r}\left(\mathbb{R}^{d_{j}}\right)$. However, an elementary scaling argument shows that a necessary condition for $(3.3)$ to hold is $r \geq(2 m-2)^{\prime}$, giving the desired contradiction.

\section{The global case}

In this section we prove Theorem 1.3.

Proof of Theorem 1.3. We let $\kappa$ dominate the $C^{1, \beta}$ norm of $F$, and note that there exists $\varepsilon>0$ such that

$$
\left|\star \bigwedge_{j=1}^{m} \star Y_{j}(\mathrm{~d} F(\omega))\right| \geq \varepsilon
$$

for all $\omega$ in the unit sphere. Using the local result in Theorem 2.1, we obtain $0<\delta<1$ depending on at most $\beta, \kappa$ and $d$, and a constant $C$ depending on at most $\varepsilon$ and $d$ satisfying

$$
\int_{U} \prod_{j=1}^{m} f_{j}\left(y_{j}\right) \delta(F(y)) \mathrm{d} y \leq C \prod_{j=1}^{m}\left\|f_{j}\right\|_{L^{(m-1)^{\prime}}\left(\mathbb{R}^{d_{j}}\right)}
$$


for all nonnegative $f_{j} \in L^{(m-1)^{\prime}}\left(\mathbb{R}^{d_{j}}\right), 1 \leq j \leq m$, where $U=B(\omega, \delta)$. By $(4.2)$, scaling, and the homogeneity of $F$,

$$
\int_{\Gamma(\omega, \delta)} \prod_{j=1}^{m} f_{j}\left(y_{j}\right) \delta(F(y)) \mathrm{d} y \leq C \sum_{k \in \mathbb{Z}} \prod_{j=1}^{m}\left\|f_{j}\right\|_{L^{(m-1)^{\prime}\left(\lambda^{k} U_{j}\right)}} .
$$

Here, $U_{j}$ is the subset of $\mathbb{R}^{d_{j}}$ given by

$$
U_{j}=\pi_{j}\left(U \cap F^{-1}(0)\right),
$$

where $\lambda=1+c \delta$, for some appropriately chosen absolute constant $c>0$, and $\pi_{j}: \mathbb{R}^{d_{1}} \times \cdots \times \mathbb{R}^{d_{m}} \rightarrow \mathbb{R}^{d_{j}}$ is the coordinate projection given by

$$
\pi_{j}\left(y_{1}, \ldots, y_{m}\right)=y_{j}
$$

Lemma 4.1. For each $\omega$ in the unit sphere, there exist $j_{1}(\omega)$ and $j_{2}(\omega)$ which are distinct and such that, for $r=1,2$, the sets $\left\{\lambda^{k} U_{j_{r}(\omega)}: k \in \mathbb{Z}\right\}$ have bounded (independent of $\omega$ ) overlap.

We remark that if one drops from $U_{j}$ the intersection with the zero set of $F$, the claimed bounded overlap property clearly ceases to hold.

Proof. We shall prove that there exists $\eta>0$ such that, for each $\omega$ in the intersection of the unit sphere with the zero set of $F$, we have $\left|\pi_{j}(\omega)\right| \geq \eta$ for at least two $1 \leq j \leq m$.

Suppose $\omega$ is on the unit sphere with $F(\omega)=0$, and suppose $\left|\pi_{j}(\omega)\right| \geq \eta$ only when $j=j_{1}$. Using the homogeneity of $F$, we have $\omega \in \operatorname{ker} \mathrm{d} F(\omega)$ and so

$$
\sum_{j=1}^{m} \pi_{j}\left(F^{(\ell)}\right) \cdot \pi_{j}(\omega)=0
$$

for each $1 \leq \ell \leq d$, where $F^{(\ell)}$ is the $\ell$ th row of $\mathrm{d} F(\omega)$. Therefore

$$
\left|\pi_{j_{1}}\left(F^{(\ell)}\right) \cdot \pi_{j_{1}}(\omega)\right| \leq C_{\kappa, m} \eta
$$

for each $1 \leq \ell \leq d$. By (4.1) it follows that

$$
\left\|\bigwedge_{k \in \mathcal{K}_{j_{1}}} \mathrm{~d} F(\omega)\left(e_{k}\right)\right\|_{\Lambda^{d_{j_{1}}\left(\mathbb{R}^{d}\right)}} \geq C_{\kappa, m} \varepsilon,
$$

which means that at least one of the components (with respect to the induced basis of $\Lambda^{d_{j_{1}}}\left(\mathbb{R}^{d}\right)$ ) of $\bigwedge_{k \in \mathcal{K}_{j_{1}}} \mathrm{~d} F(\omega)\left(e_{k}\right)$ is bounded below in magnitude by $C_{\kappa, m} \varepsilon$. This component is the determinant of a certain $d_{j_{1}} \times d_{j_{1}}$ matrix, and as a consequence, we may write

$$
\pi_{j_{1}}(\omega)=\sum_{\ell=1}^{d} \alpha_{\ell} \pi_{j_{1}}\left(F^{(\ell)}\right)
$$


for some $\alpha_{1}, \ldots, \alpha_{d} \in \mathbb{R}$ satisfying $\left|\alpha_{\ell}\right| \leq C_{\kappa, m} \varepsilon^{-1}$. Thus, by (4.3),

$$
\left|\pi_{j_{1}}(\omega) \cdot \pi_{j_{1}}(\omega)\right| \leq C_{\varepsilon, \kappa, d, m} \eta,
$$

which means that $|\omega|=\sum_{j=1}^{m}\left|\pi_{j}(\omega)\right| \leq 1 / 2$ for sufficiently small $\eta$, depending on $\varepsilon, \kappa, d$, and $m$. From this contradiction we obtain the desired conclusion.

Now

$$
\int_{\Gamma(\omega, \delta)} \prod_{j=1}^{m} f_{j}\left(y_{j}\right) \delta(F(y)) \mathrm{d} y \leq C \prod_{\substack{j \neq j_{1}(\omega), j_{2}(\omega)}}\left\|f_{j}\right\|_{L^{(m-1)^{\prime}}\left(\mathbb{R}^{d_{j}}\right)} \sum_{\substack{k \in \mathbb{Z} \\ j=j_{1}(\omega), j_{2}(\omega)}}\left\|f_{j}\right\|_{L^{(m-1)^{\prime}\left(\lambda^{k} U_{j}\right)}}
$$

and by Cauchy-Schwarz, the embedding $\ell^{(m-1)^{\prime}}(\mathbb{Z}) \subset \ell^{2}(\mathbb{Z})$, and Lemma 4.1, it follows that

$$
\int_{\Gamma(\omega, \delta)} \prod_{j=1}^{m} f_{j}\left(y_{j}\right) \delta(F(y)) \mathrm{d} y \leq C \prod_{j=1}^{m}\left\|f_{j}\right\|_{L^{(m-1)^{\prime}}\left(\mathbb{R}^{d_{j}}\right)}
$$

By compactness, the fully global estimate over $\mathbb{R}^{d_{1}} \times \cdots \times \mathbb{R}^{d_{m}}$ follows.

There is obviously some "slack" in the above argument where the embedding $\ell^{(m-1)^{\prime}}(\mathbb{Z}) \subset \ell^{2}(\mathbb{Z})$ was used. This slackness was not present in the analogous argument in [5].

\section{A. Some exterior algebra}

Lemma A.1. If $\left\{v_{1}, \ldots, v_{r}\right\} \subset \mathbb{R}^{d}$ is a linearly independent set and $\left\{n_{1}, \ldots, n_{r^{\prime}}\right\}$ is any orthonormal basis for the orthogonal complement of the space spanned by $\left\{v_{1}, \ldots, v_{r}\right\}$, where $r+r^{\prime}=d$, then

$$
\star \bigwedge_{k=1}^{r} v_{k}=\left\|\bigwedge_{k=1}^{r} v_{k}\right\|_{\Lambda^{r}\left(\mathbb{R}^{d}\right)} \bigwedge_{k=1}^{r^{\prime}} n_{k}
$$

Proof. One notes that $\left\{v_{1}, \ldots, v_{r}, n_{1}, \ldots, n_{r^{\prime}}\right\}$ is a basis for $\mathbb{R}^{d}$ and so we have the induced basis $\mathfrak{B}$ for $\Lambda^{r^{\prime}}\left(\mathbb{R}^{d}\right)$. By definition of the Hodge star we want to show that

$$
\left\|\bigwedge_{k=1}^{r} v_{k}\right\|_{\Lambda^{r}\left(\mathbb{R}^{d}\right)}\left\langle\bigwedge_{k=1}^{r^{\prime}} n_{k}, u\right\rangle_{\Lambda^{r^{\prime}}\left(\mathbb{R}^{d}\right)} \sigma=u \wedge \bigwedge_{k=1}^{r} v_{k}
$$

for each $u \in \Lambda^{n^{\prime}}\left(\mathbb{R}^{d}\right)$, where $\sigma$ is the unit basis element for $\Lambda^{d}\left(\mathbb{R}^{d}\right)$. It suffices to check (A.1) for $u \in \mathfrak{B}$ and we divide this task into the cases where $u=\bigwedge_{k=1}^{r^{\prime}} n_{k}$ and $u \neq \bigwedge_{k=1}^{r^{\prime}} n_{k}$. In the latter case is it easy to see that both sides of (A.1) are equal to zero. 
If $u=\bigwedge_{k=1}^{r^{\prime}} n_{k}$ then

$$
\begin{aligned}
& \left\|\bigwedge_{k=1}^{r} v_{k}\right\|_{\Lambda^{r}\left(\mathbb{R}^{d}\right)}\left\langle\bigwedge_{k=1}^{n^{\prime}} n_{k}, \bigwedge_{\ell=1}^{r^{\prime}} n_{\ell}\right\rangle_{\Lambda^{r^{\prime}}\left(\mathbb{R}^{d}\right)} \\
& \quad=\left\|\bigwedge_{k=1}^{r} v_{k}\right\|_{\Lambda^{r}\left(\mathbb{R}^{d}\right)} \operatorname{det}\left(n_{k} \cdot n_{\ell}\right)_{1 \leq k, \ell \leq r^{\prime}}=\left\|\bigwedge_{k=1}^{r} v_{k}\right\|_{\Lambda^{r}\left(\mathbb{R}^{d}\right)}
\end{aligned}
$$

because $\left\{n_{1}, \ldots, n_{r^{\prime}}\right\}$ is orthonormal. Now

$$
u \wedge \bigwedge_{k=1}^{r} v_{k}=\operatorname{det}\left(n_{1} \cdots n_{r^{\prime}} v_{1} \cdots v_{r}\right) \sigma
$$

and, using orthogonality considerations,

$$
\begin{aligned}
\operatorname{det}\left(n_{1} \cdots n_{r^{\prime}} v_{1} \cdots v_{r}\right)^{2} & =\operatorname{det}\left(n_{1} \cdots n_{r^{\prime}} v_{1} \cdots v_{r}\right)^{T}\left(n_{1} \cdots n_{r^{\prime}} v_{1} \cdots v_{r}\right) \\
& =\operatorname{det}\left(v_{k} \cdot v_{\ell}\right)_{1 \leq k, \ell \leq r}=\left\|\bigwedge_{k=1}^{r} v_{k}\right\|_{\Lambda^{r}\left(\mathbb{R}^{d}\right)}^{2}
\end{aligned}
$$

Hence (A.1) holds in this case too, and this completes the proof of the lemma.

\section{B. A quantitative version of the implicit function theorem}

We provide a quantitative version of the implicit function theorem for $C^{1, \beta}$ functions.

Theorem B.1. Suppose $d, n \in \mathbb{N}$ and $\beta, \kappa>0$ are given. Let $R_{1}, R_{2}>0$ be given by

$$
R_{1}=\min \left\{1, R_{2}, \frac{1}{4 d \kappa} R_{2}\right\} \quad \text { and } \quad R_{2}=\left(\frac{1}{16 d \kappa}\right)^{1 / \beta}
$$

If $F: \mathbb{R}^{n} \times \mathbb{R}^{d} \rightarrow \mathbb{R}^{d}$ is such that $\|F\|_{C^{1, \beta}} \leq \kappa, F(0,0)=0$ and

$$
\left.\mathrm{d} F(0,0)\right|_{\operatorname{span}\left\{e_{r}: n+1 \leq r \leq n+d\right\}}=I_{d},
$$

then there exists a function $\eta: B\left(0, R_{1}\right) \subset \mathbb{R}^{n} \rightarrow B\left(0, R_{2}\right) \subset \mathbb{R}^{d}$ such that

$$
F(x, \eta(x))=0 \quad \text { for each } x \text { belonging to } B\left(0, R_{1}\right) \text {, }
$$

and a constant $\widetilde{\kappa}$, depending on at most $\beta, \kappa$, and $n$, such that $\|\eta\|_{C^{1, \beta}} \leq \widetilde{\kappa}$.

For $d=1$, a proof can be found in [4]. The argument in that case extends easily to arbitrary $d$ and we omit the details. 


\section{References}

[1] Bejenaru, I. And Herr, S.: Convolutions of singular measures and applications to the Zakharov system. J. Funct. Anal. 261 (2011), no. 2, 478-506.

[2] Bejenaru, I., Herr, S., Holmer, J. and Tataru, D.: On the 2d Zakharov system with $L^{2}$ Schrödinger data. Nonlinearity 22 (2009), no. 5, 1063-1089.

[3] Bejenaru, I., Herr, S. And Tataru, D.: A convolution estimate for twodimensional hypersurfaces. Rev. Mat. Iberoam. 26 (2010), no. 2, 707-728.

[4] Bennett, J. And Bez, N.: Some nonlinear Brascamp-Lieb inequalities and applications to harmonic analysis. J. Funct. Anal. 259 (2010), no. 10, 2520-2556.

[5] Bennett, J., Bez, N. And Gutiérrez, S.: Global nonlinear Brascamp-Lieb inequalities. J. Geom. Anal., DOI : 10.1007/s12220-012-9307-3.

[6] Bennett, J., Carbery, A., Christ, M. And TaO, T.: The Brascamp-Lieb inequalities: finiteness, structure and extremals. Geom. Funct. Anal. 17 (2007), no. $5,1343-1415$.

[7] Bennett, J., Carbery, A. And TaO, T.: On the multilinear restriction and Kakeya conjectures. Acta Math. 196 (2006), no. 2, 261-302.

[8] Bennett, J., Carbery, A. And Wright, J.: A non-linear generalisation of the Loomis-Whitney inequality and applications. Math. Res. Lett. 12 (2005), no. $4,443-457$.

[9] Moyua, A., Vargas, A. And Vega, L.: Restriction theorems and maximal operators related to oscillatory integrals in $\mathbb{R}^{3}$. Duke Math. J. 96 (1999), no. 3, 547-574.

[10] Ruiz, A. And Vargas, A.: Partial recovery of a potential from backscattering data. Comm. Partial Differential Equations 30 (2005), no. 1-3, 67-96.

[11] Stovall, B.: $L^{p}$ improving multilinear Radon-like transforms. Rev. Mat. Iberoam. 27 (2011), no. 3, 1059-1085.

[12] TAO, T.: Multilinear weighted convolution of $L^{2}$-functions, and applications to nonlinear dispersive equations. Amer. J. Math. 123 (2001), no. 5, 839-908.

[13] TaO, T., Vargas, A. And Vega, L.: A bilinear approach to the restriction and Kakeya conjectures. J. Amer. Math. Soc. 11 (1998), no. 4, 967-1000.

[14] Tao, T. And Wright, J.: $L^{p}$ improving bounds for averages along curves. J. Amer. Math. Soc. 16 (2003), no. 3, 605-638.

Received September 13, 2011; revised September 19, 2012.

Jonathan Bennett: School of Mathematics, University of Birmingham, Edgbaston, Birmingham, B15 2TT, England.

E-mail: J.Bennett@bham.ac.uk

NeAl Bez: School of Mathematics, University of Birmingham, Edgbaston, Birmingham, B15 2TT, England.

E-mail: N.Bez@bham.ac.uk

Susana Gutiérrez: School of Mathematics, University of Birmingham, Edgbaston, Birmingham, B15 2TT, England.

E-mail: S.Gutierrez@bham.ac.uk 